\title{
A Tribute to the "Naughty" Follicular Epithelium
}

\author{
K. S. Gopinath ${ }^{1} \cdot$ Abhay K. Kattepur ${ }^{2}$ \\ Published online: 21 February 2022 \\ (c) The Author(s), under exclusive licence to Indian Association of Surgical Oncology 2022
}

The thyroid follicle forms the structural and functional unit of the thyroid gland. Each follicle consists of a lining of the follicular epithelium which surrounds a thyroglobulin pool. The follicle is central to the homeostatic functioning of the thyroid, whereby signals from the hypothalamo-pituitary axis are relayed to the effector tissues through these cells. The activity of the thyroid gland is mainly important for metabolism, growth and development of the human body.

Pathological changes in the follicular epithelium lead to the development of both thyroid nodules and thyroid cancers. Thyroid nodules are increasingly being diagnosed, owing to the rampant use of radiological investigations performed for various disease states. Management of thyroid nodules requires a dedicated, multidisciplinary team of specialists not limited to surgeons, endocrinologists, radiologists and nuclear medicine specialists.

Cancers arising from this follicular epithelial lining have distinct clinical, pathological and prognostic features, giving rise to a spectrum of tumours: from the biologically indolent classical papillary thyroid carcinomas to the highly lethal anaplastic thyroid carcinomas. This has resulted in the development of molecular theranostics, playing an important role in the treatment armamentarium. Thyroid carcinogenesis is a complex multistep process involving various growth factors, oncogenes and angiogenetic and cell cycle pathways, including the dysregulation in microRNA and epigenetic processes. This has been depicted in the diagram given below and has paved the way for the development of molecular markers for prognostication and treatment (in the form of systemic targeted therapy in advanced thyroid cancers).

The "naughtiness" of the follicular epithelium stems from a clinical perspective, underlined by the fact that (a) whether every thyroid cancer needs to be treated or not; (b) if so, the extent of treatment needs to be individualized in every case; and (c) the treatment algorithms in the management of microcarcinomas remain controversial to date.

The follicular epithelium behaves biologically differently in different parts of the globe. Incidentalomas in the West to locally advanced and disseminated cancers in our subcontinent are usual clinical scenarios. Furthermore, rare sites of dissemination to the acral bones, retina etc. have also been reported and treated with satisfactory outcomes. There is a need to develop a global thyroid cancer databank to address many of the problems of thyroid nodules and cancers alike.

This current issue highlights the surgical management of thyroid cancers from hemithyroidectomy to total

K. S. Gopinath

ijso@iasoindia.in

1 RL Jalappa Institute of Oncology, SDUAHER, Tamaka, Kolar, India

2 Department of Surgical Oncology, RL Jalappa Institute of Oncology, SDUAHER, Tamaka, Kolar, India 
thyroidectomy and resection of adjacent organs/viscera in aggressive tumours. The usefulness of radioiodine has also been highlighted. The role of systemic treatment in the management of metastatic disease including targeted therapy has been presented. The Indian experience from different centres have been included.

I thank all the authors and their team, reviewers and my editorial board in bringing out this issue on thyroid and parathyroid diseases.

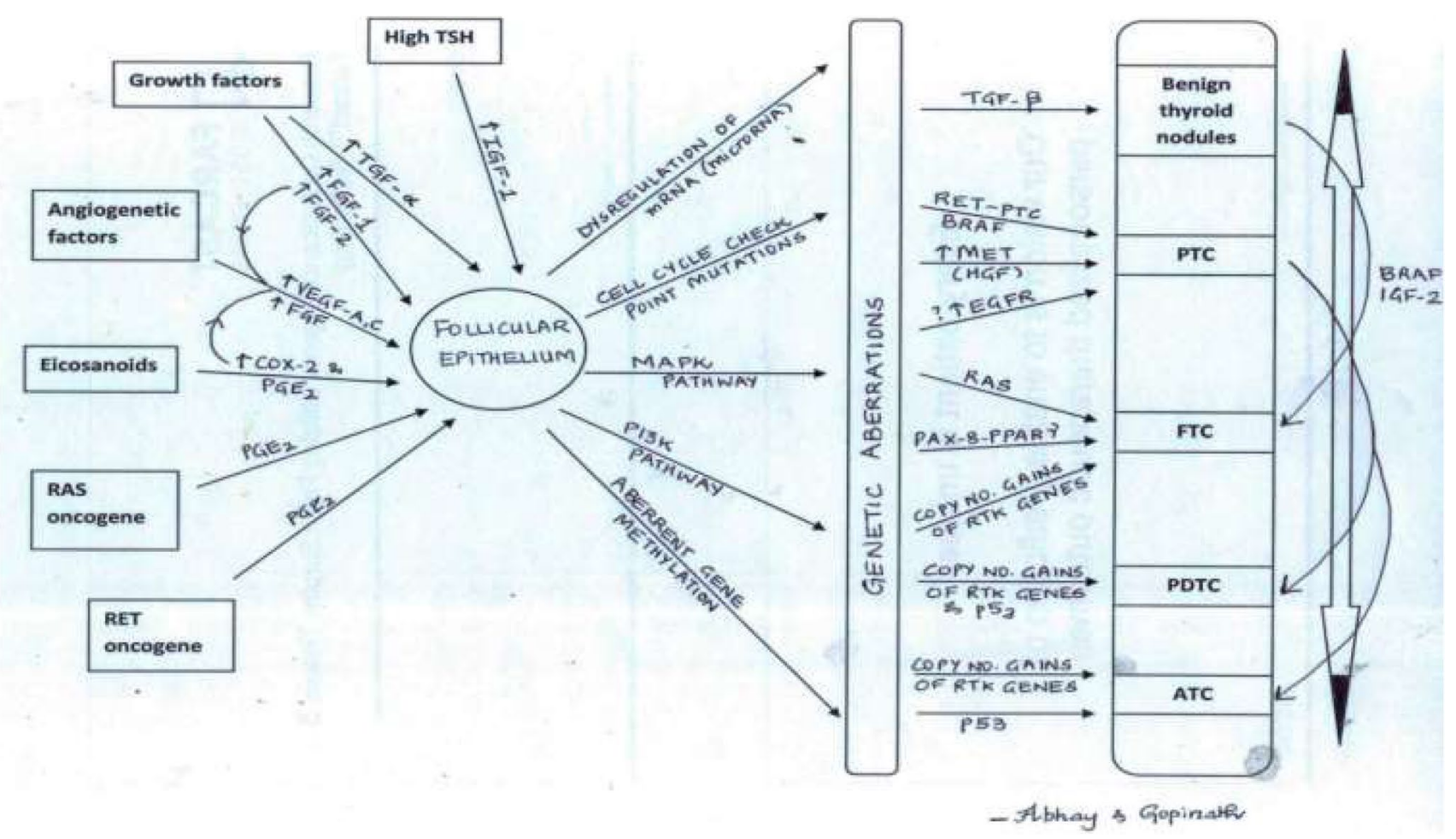

Publisher's Note Springer Nature remains neutral with regard to jurisdictional claims in published maps and institutional affiliations. 